\title{
Comparison of four commercial lgM and IgG ELISA kits for diagnosing brucellosis
}

Correspondence

Robyn A. Stoddard

rastoddard@cdc.gov or

frd8@cdc.gov

Received 13 April 2011

Accepted 8 August 2011

\author{
Moustafa Abdel Fadeel, ${ }^{1}, 2$ Alex R. Hoffmaster, ${ }^{1}$ Jianrong Shi, ${ }^{1}$ \\ Guillermo Pimentel $^{2}$ and Robyn A. Stoddard ${ }^{1}$
}

\author{
${ }^{1}$ National Center for Emerging and Zoonotic Infectious Diseases, Centers for Disease Control and \\ Prevention, 1600 Clifton Road NE, Atlanta, GA 30333, USA \\ ${ }^{2}$ Global Disease Detection and Response Program (GDDRP), US Naval Medical Research Unit \\ No. 3 (NAMRU-3), PSC 452, Box 5000, FPO AE 09835-0007, Cairo, Egypt
}

\begin{abstract}
Brucellosis is a worldwide zoonotic disease that often requires serology for diagnosis. The serum agglutination test is the gold standard assay, but ELISAs are used by many laboratories. Many commercial ELISAs are available, but few studies have compared their performance. This study compared the ability of four commercially available ELISA kits (from Bio-Quant, ImmunoBiological Laboratories - America, Vircell and Euroimmun) to diagnose brucellosis in patients from Egypt and the USA. The sensitivities for all kits tested, except the Vircell kit, were $>90 \%$, whilst the specificities were variable, with the Bio-Quant assay having a specificity of $<40 \%$.

Detection of $\operatorname{lgG}$ antibody was more sensitive than detection of $\lg M$ antibody for diagnosing brucellosis cases, but the specificity was comparable. Overall, there was good agreement between all of the kits except for the Bio-Quant kit. None of the diagnostic assays was $100 \%$ reliable for diagnosing brucellosis; therefore, serology results need to be considered in tandem with patient history, clinical signs and other test results.
\end{abstract}

\section{INTRODUCTION}

Brucellosis is a zoonotic disease that is found worldwide and is usually caused by one of four species of Brucella (Brucella abortus, Brucella melitensis, Brucella suis and Brucella canis), which can be transmitted to humans from cattle, goats and sheep, swine and dogs, respectively (Al Dahouk et al., 2003; Young, 1995). Clinical signs of brucellosis in humans are non-specific, and routine laboratory tests are not able to diagnose the disease. Instead, specific testing for Brucella is required for diagnosis by demonstration of the organism or of antiBrucella antibodies (Araj, 1999; Young \& Corbel, 1989; Young, 1995). There are many serological methods that can be used for the detection of antibodies to diagnose brucellosis, such as a serum agglutination test (SAT), Rose Bengal test, complement fixation, indirect Coombs' test and ELISAs. The SAT can be run as either a serum tube agglutination test (STA) or microplate serum agglutination test (MAT). It is the most popular diagnostic assay and the gold standard assay of comparison for other serological tests (Al Dahouk et al., 2003; Araj, 1999; Pappas et al., 2005; Young, 1995). The SAT is considered the best serological assay for diagnosing acute, uncomplicated cases,

Abbreviations: AFI, acute febrile illness; CDC, Centers for Disease Control and Prevention; $\mathrm{Cl}$, confidence interval; IBL, Immuno-Biological Laboratories; MAT, microplate serum agglutination test; SAT, serum agglutination test; STA, serum tube agglutination test. but it can yield false positives in chronic or complicated cases and can also be time-consuming for running large numbers of samples (Al Dahouk et al., 2003; Araj, 1999). ELISAs can overcome some of the negative aspects of the SAT (Pappas et al., 2005). The reported sensitivity and specificity of ELISAs varies in the literature and can be influenced by the individual assay used and the location of the study (Al Dahouk et al., 2003; Araj, 1999). In the USA, commercial diagnostic laboratories rely on ELISA kits, and confidence in results from different commercial laboratories can be challenging due to the use of the different ELISA kits and the lack of published studies comparing kits. The purpose of this study was to compare the ability of four commercially available IgG and IgM ELISA kits to diagnose brucellosis using sera from patients in Egypt and the USA.

\section{METHODS}

Case sera. A total of 372 serum samples were used from 186 casepatients from studies published in the USA (Buchanan \& Faber, 1980) and Egypt (Afifi et al., 2005). Specific clinical or treatment information was not available for these case-patients. Serum samples were collected from acutely ill patients at admission (acute sample) and 27 months after antimicrobial therapy was initiated (convalescent sample). Case-patients were diagnosed with brucellosis in one of the two following categories based on the presence of clinical signs of brucellosis and diagnostic results: (i) culture positive for and with an 
appropriate antibody response against a Brucella spp. or (ii) culture negative but with the presence of high antibody titres or seroconversion. An STA was originally run on all samples with a cut-off titre of $\geqslant 160$ for positive results and $20-80$ for borderline results, with $<20$ considered negative for samples from the USA. Samples from Egypt with a titre of $\geqslant 320$ were considered positive and with a titre of $<320$ were considered negative (Al Dahouk et al., 2003). All serum samples were retested at the Centers for Disease Control and Prevention (CDC) with a MAT to confirm the original tube agglutination results.

Samples from the USA were collected during an outbreak of brucellosis that occurred at a meatpacking plant in Iowa (Buchanan \& Faber, 1980). A total of 119 case-patients were included from this outbreak. Of the 119 case-patients, 93 were originally diagnosed through serology, and the remaining 26 case-patients were positive by both serology and culture for brucellosis. B. abortus was cultured from 24 of the patients and B. suis from 2 patients. The mean time between acute and convalescent samples was 143 days $(\mathrm{SD}=79$ days).

Samples from Egypt were collected during an acute febrile illness (AFI) surveillance study (Afifi et al., 2005). There was a total of 67 case-patients from Egypt, 37 diagnosed through serology, and the remaining 30 case-patients being both serologically positive and culture positive for $B$. melitensis. The mean time between acute and convalescent samples was 153 days $(\mathrm{SD}=40$ days $)$.

Control sera. A total of 183 single control specimens were obtained from healthy controls and patients in the USA and Egypt. Of the 183 control specimens, 87 were from healthy controls (USA and Egypt) and 50 were from patients with AFI of unknown origin (Egypt) (Afifi et al., 2005). AFI patients were culture negative for Brucella spp. and serologically negative when tested with SAT initially. As with the case sera, all serum samples were retested at the CDC with the MAT to confirm the original results. The remaining 46 samples were from patients in the USA and Egypt confirmed with diseases caused by the following aetiological agents: Escherichia coli O157 $(n=6)$, Vibrio cholerae $(n=5)$, Francisella tularensis $(n=5)$, Salmonella enterica serovar Typhi $(n=10)$, Rickettsia typhi $(n=10)$ and Leptospira interrogans $(n=10)$.

MAT. Serum samples were tested at the CDC by MAT, the gold standard for serological diagnosis at the CDC (Brown et al., 1981), with minor modifications including the use of U-bottomed plates, incubation at $28{ }^{\circ} \mathrm{C}$ and discontinued use of safranin. The results were interpreted as described for the STA.

Commercial ELISA kits. All testing was performed at the CDC. The following kits were evaluated in this study: Bio-Quant Brucella IgM ELISA and IgG ELISA, Immuno-Biological Laboratories (IBL) America Brucella IgM ELISA and IgG ELISA, Vircell Brucella ELISA IgM and ELISA IgG, and Euroimmun anti-Brucella abortus IgM ELISA and IgG ELISA. ELISA kits were run and interpreted according to the manufacturers' procedures and recommendations. The choice of ELISA kits tested in this study does not reflect endorsement by the CDC.

Data analysis. Sensitivity and specificity values were calculated for the IgG and IgM tests separately and together. For the combined IgM and $\operatorname{IgG}$ sensitivity and specificity calculations, test results were interpreted as follows: positive, if one or both of $\operatorname{IgG}$ or $\operatorname{IgM}$ was positive; borderline, if both IgG and IgM were borderline, or one was borderline and the other negative; and negative, if both IgG and IgM were negative. Unless otherwise stated, discussions of sensitivity and specificity data refer to the combined (IgM $+\operatorname{IgG})$ sensitivity and specificity results. Confidence intervals (CIs) were also calculated using the standard normal distribution for proportions. Student's $t$ test was used to determine whether there was a significant difference in the time between acute and convalescent samples for samples from case-patients in Egypt and the USA, and $P<0.05$ was considered to be statistically significant. The $\kappa$ value was calculated to measure the degree of agreement between any two assays in classifying an individual sample correctly. A $\kappa$ value of $\geqslant 0.75$ indicated excellent agreement between assays, whilst a value of $<0.40$ indicated poor agreement (Bajani et al., 2003; Fleiss, 1981; Sim \& Wright, 2005).

\section{RESULTS AND DISCUSSION}

\section{Sensitivity and specificity of ELISA kits (IgM and IgG combined)}

Three of the four commercial ELISA kits were highly sensitive, ranging from 98.5 to $100 \%$ when looking at paired sera (Table 1). The Vircell ELISA kit performed well on sera from Egypt, with a sensitivity of $>95 \%$ for paired and acute-phase sera, but with a low sensitivity for paired $(79.0 \%)$, acute-phase $(72.3 \%)$ and convalescent $(70.6 \%)$ sera from patients in the USA (Table 1). We observed a $>99.0 \%$ sensitivity for paired sera with the IBL kit in our study, whilst another study conducted by Cakan et al. (2008) reported the IBL kit to have a $93.1 \%$ sensitivity when testing samples from Turkey.

The sensitivity of the ELISA kits for convalescent-phase sera from patients in Egypt, ranging from 85.1 to $100 \%$, was higher than the sensitivity of the MAT (65.7\%) (Table 1). Similar results were seen with convalescent-phase samples from patients in the USA, with all kits except the Vircell ELISA being more sensitive than the MAT (Table 1). These results corroborate other reports that ELISAs are better at detecting subacute and chronic brucellosis than the MAT because agglutination tests have the potential for negative results much sooner after infection compared with ELISAs, especially those detecting IgG (Al Dahouk et al., 2003; Araj et al., 1986a; Gotuzzo et al., 1986; Young \& Corbel, 1989). We also found increased variation between the sensitivities of the kits when comparing convalescentphase sera from patients in Egypt versus the USA (Table 1). The MAT, IBL and Euroimmun ELISA kits were less sensitive when testing convalescent sera from Egypt, whilst the Vircell kit was more sensitive when testing convalescent samples from Egypt $(P<0.05)$. Timing from when acute and convalescent samples were taken was not found to be statistically significant $(P=0.29)$, as the mean number of days was 153 for sampling of patients from Egypt and 143 for sampling of patients from the USA. One potential explanation for the higher sensitivity of convalescent samples from the USA could be because these samples were from patients with occupational exposure who may have experienced continued exposure if they were still working, prolonging the production of antibody (Al Dahouk et al., 2003).

The specificities of the commercial ELISA kits were higher when testing samples from Egypt compared with the USA, but the difference in performance was only statistically significant for the Bio-Quant kit (Table 1). For sera from Egypt, the specificities of the ELISA kits ranged from 
Table 1. Sensitivity and specificity of combined IgM and IgG for four commercial ELISAs and MAT for detection of Brucella antibodies in patients in different geographical areas calculated using $2 \times 2$ contingency tables

Results in bold indicate a statistically significant difference $(P<0.05)$ in sensitivity or specificity in Egypt versus the USA.

\begin{tabular}{|c|c|c|c|c|}
\hline \multirow[t]{2}{*}{ Country/assay } & \multicolumn{3}{|c|}{ Percentage sensitivity (95\% CI) } & \multirow{2}{*}{$\begin{array}{l}\text { Percentage specificity } \\
\qquad(95 \% \mathrm{CI})\end{array}$} \\
\hline & Paired sera ${ }^{\star}$ & Acute-phase sera & Convalescent-phase sera & \\
\hline MAT & $98.5(92.0-100.0)$ & $98.5(92.0-100.0)$ & $65.7(53.1-76.9)$ & $100.0(97.5-100.0)$ \\
\hline Bio-Quant & $100.0(94.6-100.0)$ & $100.0(94.6-100.0)$ & $100.0(94.6-100.0)$ & $34.5(26.8-42.8)$ \\
\hline IBL & $100.0(94.6-100.0)$ & $100.0(94.6-100.0)$ & $88.1(77.8-94.7)$ & $98.6(95.1-99.8)$ \\
\hline Euroimmun & $100.0(94.6-100.0)$ & $100.0(94.6-100.0)$ & $86.6(76.0-93.7)$ & $89.7(83.5-94.1)$ \\
\hline USA $(n)$ & 238 & 119 & 119 & 38 \\
\hline MAT & $100.0(97.0-100.0)$ & $100.0(97.0-100.0)$ & $80.7(72.4-87.3)$ & $68.4(51.4-82.5)$ \\
\hline Bio-Quant & $100.0(97.0-100.0)$ & $100.0(97.0-100.0)$ & $99.2(95.4-100.0)$ & $10.5(2.9-24.8)$ \\
\hline IBL & $99.2(95.4-100.0)$ & $99.2(95.4-100.0)$ & $98.3(94.1-99.8)$ & $97.4(86.2-99.9)$ \\
\hline Vircell & $79.0(70.6-85.9)$ & $72.3(63.3-80.1)$ & $70.6(61.5-78.6)$ & $97.4(86.2-99.9)$ \\
\hline
\end{tabular}

${ }^{\star}$ For any assay, a case was considered positive if the acute and/or the convalescent $\operatorname{specimen}(\mathrm{s})$ tested positive.

$34.5 \%$ (Bio-Quant) to $98.6 \%$ (IBL and Vircell), with all of the kits except the Bio-Quant kit having a specificity of $>80 \%$ (Table 1). A study conducted by Cakan et al. (2008) reported a specificity of $98.2 \%$ for the IBL ELISA, similar to what was found in this study. For samples from the USA, the specificities of the commercial ELISA kits were highly variable, ranging from $10.5 \%$ (Bio-Quant) to $97.4 \%$ (IBL and Vircell) (Table 1).

The results for cross-reactivity of the assays tested can be seen in Table 2. Of the patients with a confirmed diagnosis, cross-reactivity was most commonly seen when testing samples from patients with E. coli O157 (Table 2). When testing undiagnosed AFI sera, neither the IBL nor the Vircell kit had any false-positive results, whilst $64.0 \%$ of the Bio-Quant results were false positives (Table 2). The IBL, Vircell and Euroimmun ELISA kits had false-positive reactions to $<10 \%$ of the sera tested from healthy patients, with the Bio-Quant ELISA reacting to $71.3 \%$ of the samples (Table 2). Over $70 \%$ of samples tested with the Bio-Quant ELISA kits had false-positive reactions, with all specimen categories being represented (Table 2). The Euroimmun ELISA kits were cross-reactive with $12.0 \%$ of the samples tested, with false-positive reactions being seen with sera from patients in all of the categories tested except for $L$. interrogans (Table 2). Both the IBL and Vircell ELISA kits showed minimal cross-reactivity (Table 2).

Potential explanations for variable and low sensitivity and/ or specificity for the various kits could be human error in testing or data input, bias of sample selection, sample size, misdiagnosis of original disease or co-infection, and variation among the reagents used for making the kits. The Bio-Quant ELISA showed very low specificities for both Egypt (34.5\%) and the USA (10.5\%); however, the
ELISA was 100 and $99.2 \%$ sensitive for paired samples from patients from Egypt and the USA, respectively (Table 1 ), suggesting the kits may be sacrificing specificity in order to be highly sensitive. When testing sera from 87 healthy individuals using the Bio-Quant ELISA, $71.3 \%(n=62)$ of the samples were found to be borderline or positive, much higher than with any of the other assays tested (Table 2). Of the 22 serum samples collected from healthy persons in the USA, $19(86.4 \%)$ were borderline or positive, and 43 of the 65 serum samples $(66.2 \%)$ from healthy persons in Egypt were borderline or positive (data not shown). These results showed that the positive results seen with the Bio-Quant ELISA were not likely to be the result of residual antibodies present after previous infections in persons living in a country such as Egypt that is highly endemic for brucellosis. Another factor that may have influenced the specificity of assays when testing samples from the USA was that the sample size was much smaller than for Egypt (38 and 145, respectively) (Table 1). Additionally, we only had sera available from Egypt for testing patients with AFI of unknown origin (Table 2).

There is no standardization of reagents that are used in ELISAs for detecting anti-Brucella antibodies in humans, and the type and quality of reagents can have a large effect on the performance of an assay (Al Dahouk et al., 2003; Araj et al., 1990). The IBL and Euroimmun ELISA kits use a bacterial lysate of $B$. abortus strain W99 as the antigen. The IBL ELISA kits had a specificity of $>97 \%$ for sera tested from both Egypt and the USA, whilst the Euroimmun kits had a lower specificity for the samples from Egypt (89.7\%) and the USA (81.6\%) (Table 1). Although the Vircell ELISA kits use a different antigen from the IBL kits (LPS of $B$. abortus strain S-99), they had the same specificity as the IBL kits for sera tested from both 
Table 2. Cross-reactivity of six Brucella serology assays with serum specimens from patients in Egypt and the USA who were previously diagnosed with AFI of unknown origin or other infections

Results for the ELISA kits were considered positive if one or both $\operatorname{IgG} / \operatorname{IgM}$ was positive, borderline if both $\operatorname{IgG} / \operatorname{IgM}$ were borderline or one was borderline and the other negative, and negative if both $\operatorname{IgG} / \operatorname{IgM}$ were negative.

\begin{tabular}{|c|c|c|c|c|c|c|}
\hline \multirow[t]{2}{*}{ Category } & \multirow{2}{*}{$\begin{array}{c}\text { Total no. of } \\
\text { specimens tested }\end{array}$} & \multicolumn{5}{|c|}{ No. (\%) of specimens positive or borderline by: } \\
\hline & & MAT & Bio-Quant & IBL & Vircell & Euroimmun \\
\hline E. coli $\mathrm{O} 157^{\star}$ & 6 & $5(83.3)$ & $6(100)$ & $1(16.7)$ & $1(16.7)$ & $2(33.3)$ \\
\hline V. cholerae $^{*}$ & 5 & $3(60.0)$ & $4(80.0)$ & $0(0)$ & $0(0)$ & $3(60.0)$ \\
\hline F. tularensis ${ }^{*}$ & 5 & $1(20.0)$ & $5(100)$ & $0(0)$ & $0(0)$ & $1(20.0)$ \\
\hline $\begin{array}{l}\text { S. enterica serovar } \\
\text { Typhi } \dagger\end{array}$ & 10 & $0(0)$ & $9(90.0)$ & $0(0)$ & $0(0)$ & $1(10.0)$ \\
\hline R. typhi† & 10 & $0(0)$ & $7(70.0)$ & $0(0)$ & $1(10.0)$ & $2(20.0)$ \\
\hline L. interrogans $\dagger$ & 10 & $0(0)$ & $4(40.0)$ & $0(0)$ & $0(0)$ & $0(0)$ \\
\hline $\begin{array}{l}\text { AFI of unknown } \\
\text { origin } \dagger\end{array}$ & 50 & $0(0)$ & $32(64.0)$ & $0(0)$ & $0(0)$ & $6(12.0)$ \\
\hline $\begin{array}{l}\text { Healthy control } \\
\text { patients } \ddagger\end{array}$ & 87 & $3(3.4)$ & $62(71.3)$ & $2(2.3)$ & $1(1.1)$ & $7(8.0)$ \\
\hline Total & 183 & $12(6.6)$ & $129(70.5)$ & $3(1.6)$ & $3(1.6)$ & $22(12.0)$ \\
\hline
\end{tabular}

${ }^{\star}$ Specimens from patients in the USA.

$\dagger$ Specimens from patients in Egypt.

$\ddagger$ Specimens from patients in the USA and Egypt.

Egypt and the USA, although they had a lower sensitivity (Table 1). The Bio-Quant kits, with the lowest specificity of all kits evaluated, use $B$. abortus outer membrane (strain not identified) as the antigen, which consists primarily of LPS and outer-membrane proteins (Young \& Corbel, 1989). Several studies have shown that serology assays using LPS as the antigen have the potential to cross-react with other bacteria, reducing the specificity of the assay (Mantecón et al., 2006), whilst others state that assays using LPS should have better specificity (Sharma et al., 2008). For serological assays that use whole cells as their antigen, LPS is also very important for detecting antibody, and all the kits had LPS purified or as part of the whole cell. This suggests that the manner in which the antigen is handled may have an influence on assay performance (Mantecón et al., 2006; Young \& Corbel, 1989).

\section{Sensitivity and specificity of IgM and IgG ELISA kits}

In addition to determining the sensitivity and specificity of the combined results of the IgM and IgG ELISA kits, we determined the sensitivity and specificity of the IgM and IgG ELISA kits individually for each of the manufacturers. Detection of the IgG antibody class was found to be more sensitive $(P<0.05)$ than IgM detection when using paired, acute or convalescent sera, with a statistically significant difference for all ELISA kits except for those from Euroimmun (paired and acute-phase sera) and Vircell (acute-phase sera) (Table 3). When testing acute-phase sera, the Euroimmun IgM ELISA was found to be more sensitive than the IgG ELISA (Table 3). The specificities of the IgM versus IgG kits were comparable for the IBL and Vircell kits; however, there was a statistically significant difference $(P<0.05)$ between the IgM and IgG specificities for the Bio-Quant and Euroimmun kits (Table 3). The BioQuant ELISA had a higher specificity when detecting the IgM antibody class, whilst the Euroimmun kit had a higher specificity when detecting the IgG antibody class (Table 3 ). The specificity for the IgG antibody class ranged between $44.8 \%$ (Bio-Quant) and $100 \%$ (Vircell), and for the IgM antibody class between 69.4\% (Bio-Quant) and 99.5\% (IBL) (Table 3).

The higher sensitivity of IgG ELISA compared with IgM ELISA found in the current study has been demonstrated by Mantecón et al. (2006). IgM antibodies are produced in the first week after infection and IgG antibodies increase during the second week, whilst symptoms of disease can occur after 2-3 weeks, and both IgM and IgG classes peak about 1 month after infection (Al Dahouk et al., 2003). IgG antibodies are also considered to be a better indicator of active brucellosis infection than IgM, because IgM persists in the absence of clinical signs, whilst IgG is present only in symptomatic cases (Buchanan \& Faber, 1980). The titres for IgM and IgG for all case-patients were already elevated in acute-phase samples, suggesting that an immune reaction had already been mounted. This has been seen in other studies in patients with both acute and subacute brucellosis (Araj et al., 1986a, 1990). The higher sensitivity of the IgG ELISA kits in the USA could be explained by the fact that these individuals were abattoir workers who may 
Table 3. Comparison of sensitivity $(n=186)$ and specificity $(n=183)$ for $\lg$ G versus lgM tests for the four commercial Brucella ELISA kits

Results in bold indicate a statistically significant difference $(P<0.05)$ in sensitivity or specificity between $\operatorname{IgG}$ and $\operatorname{IgM}$.

\begin{tabular}{|c|c|c|c|c|c|c|c|c|}
\hline \multirow[t]{2}{*}{ Assay } & \multicolumn{6}{|c|}{ Percentage sensitivity } & \multicolumn{2}{|c|}{ Percentage specificity } \\
\hline & \multicolumn{2}{|c|}{ Paired sera $^{*}$} & \multicolumn{2}{|c|}{ Acute-phase sera } & \multicolumn{2}{|c|}{ Convalescent-phase sera } & IgG & IgM \\
\hline Bio-Quant & 100 & 95.2 & 100 & 95.2 & 99.5 & 79.6 & 44.8 & 69.4 \\
\hline IBL & 98.9 & 48.4 & 98.9 & 44.1 & 93.6 & 13.4 & 98.4 & 99.5 \\
\hline
\end{tabular}

${ }^{\star}$ For any assay, a case was considered positive if the acute and/or convalescent specimen(s) tested positive.

have had elevated IgG levels due to a continued occupational exposure to Brucella species (Al Dahouk et al., 2003). Other studies support the findings that IgG is significantly more sensitive than $\operatorname{IgM}$ for convalescentphase samples due to the fact that IgM levels decrease faster than IgG levels (Mantecón et al., 2006; Marrodan et al., 2001).

\section{Agreement between assays}

Despite the variability in sensitivity and specificity, three of the four ELISA kits (IBL, Vircell and Euroimmun) tested had good agreement with the MAT results and with each other, evidenced by $\kappa$ values of $\geqslant 0.75$ (Table 4 ). The MAT and IBL ELISA kit showed the greatest agreement, with a $\kappa$ correlation of 0.95 (95\% CI 0.92-0.97; Table 4). The BioQuant ELISA kit showed poor agreement with all assays tested, with $\kappa$ values ranging between 0.32 and 0.37 (Table 4), and was the only ELISA in this study that used outer membrane as its source of antigen. The Bio-Quant kit had a high sensitivity in this study, but the low specificity resulted in the low correlation in comparison with the other assays.

\section{Practical and economic comparisons of the assays}

In addition to the good agreement between the results of the commercial ELISA kits, they each required similar serum volumes $(10-20 \mu \mathrm{l})$ and less time $(2.5-3.5 \mathrm{~h})$ to complete the assay than the MAT (Table 5). The commercial ELISA kits ranged in price from $\mathfrak{E} 2.21$ to 4.68 (US\$3.40 to 7.20) per sample, with the IBL kit being the most expensive kit (Table 5).

\section{Conclusions}

In this study, four commercial IgM and IgG ELISA kits were tested on samples from case-patients previously diagnosed through culture and/or MAT to evaluate their

Table 4. Agreement between diagnostic assays for brucellosis in classifying cases as positive and controls as negative

\begin{tabular}{|lccc|}
\hline Assays compared & $\begin{array}{c}\text { No. (\%) cases testing positive } \\
(\boldsymbol{n}=\mathbf{1 8 6})^{\star}\end{array}$ & $\begin{array}{c}\text { No. (\%) controls testing negative } \\
(\boldsymbol{n}=\mathbf{1 8 3})\end{array}$ & $\boldsymbol{\kappa}$ correlation $(\mathbf{9 5} \% \mathbf{C I}) \dagger$ \\
\hline MAT and Bio-Quant & $185(99.5)$ & $52(28.4)$ & $0.37(0.30-0.44)$ \\
MAT and IBL & $184(98.9)$ & $169(92.4)$ & $0.95(0.92-0.97)$ \\
MAT and Vircell & $159(85.5)$ & $169(92.4)$ & $0.88(0.84-0.91)$ \\
MAT and Euroimmun & $185(99.5)$ & $152(83.1)$ & $0.90(0.86-0.93)$ \\
Bio-Quant and IBL & $185(99.5)$ & $54(29.5)$ & $0.37(0.30-0.44)$ \\
Bio-Quant and Vircell & $160(86.0)$ & $54(29.5)$ & $0.32(0.26-0.39)$ \\
Bio-Quant and Euroimmun & $186(100.0)$ & $49(26.8)$ & $0.36(0.29-0.44)$ \\
IBL and Vircell & $159(85.5)$ & $178(97.3)$ & $0.91(0.88-0.94)$ \\
IBL and Euroimmun & $185(99.5)$ & $158(86.3)$ & $0.90(0.86-0.94)$ \\
Vircell and Euroimmun & $160(86.0)$ & $160(87.4)$ & $0.84(0.79-0.88)$ \\
\hline
\end{tabular}

*For each assay, a case was considered positive if the acute and/or the convalescent-phase specimen(s) tested positive.

$\dagger \mathrm{A} \kappa$ correlation of $\geqslant 0.75$ was considered a good agreement, a correlation of $0.40-0.74$ was considered a fair agreement, and a correlation of $<0.40$ was considered a poor agreement. 
Table 5. Comparison of the serum volume needed, assay time and cost of running the five serological assays for diagnosing brucellosis

\begin{tabular}{|lclccc|}
\hline Factor & MAT & Bio-Quant & IBL & Vircell & Euroimmun \\
\hline $\begin{array}{l}\text { Serum volume needed } \\
(\mu \mathrm{l})\end{array}$ & 40.0 & 20.0 & 10.0 & 10.0 & 20.0 \\
Assay time $(\mathrm{h})^{*}$ & $21.0^{*}$ & $2.5 \dagger$ & $3.5 \dagger$ & $3.0 \dagger$ & $2.75 \dagger$ \\
Cost per serum & $0.91[1.40]$ & $2.86[4.40]$ & $4.68[7.20]$ & $2.21[3.40]$ & $2.86[4.40]$ \\
sample $(\mathfrak{E})[\mathrm{US} \$]$ & & & & \\
\hline
\end{tabular}

*Time needed to set up 61 samples by one person to test for total and IgG antibody titres.

$\dagger$ Time needed to test one ELISA plate (96 tests) for both IgM and IgG antibody by one person, including setting up and incubation time for each assay.

ability to diagnose brucellosis in humans in Egypt and the USA. Three of the four commercial kits tested had sensitivities of $>90 \%$, with the Vircell ELISA kit being the exception when considering results of paired or acutephase sera. When testing convalescent sera, the ELISAs were more sensitive than the MAT. Most of the ELISAs had a higher sensitivity for IgG antibody detection than IgM antibody, but specificity was comparable when comparing IgM versus IgG detection. Overall, there appeared to be good agreement between all of the assays except for the Bio-Quant ELISA kit.

Although the MAT is the recommended assay in the routine diagnosis of acute brucellosis (Araj et al., 1986b) and is the test required by the Council of State and Territorial Epidemiologists in the USA (CDC, 1997), ELISAs are used in many commercial laboratories. This study has shown that there is good correlation between the various ELISA kits available and that sensitivity for diagnosing brucellosis is increased when $\operatorname{IgM}$ and $\operatorname{IgG}$ results are considered together. Clinicians are recommended to enquire with testing laboratories as to which assay is being used and who the manufacturer of the assay is when considering test results. Whilst these assays generally exhibited good sensitivity and specificity, serology results need to be considered in tandem with patient history, clinical signs and other test results.

\section{ACKNOWLEDGEMENTS}

The authors would like to thank Drs Luke Faber, Thomas Buchanan and Arnold Kaufmann for conducting the original study that provided samples from the USA. We would also like to thank Dr Mary Ari for her assistance in obtaining sera and also her input into the study; Dr Deborah Talkington, Jim Pruckler and Christopher Sexton for providing sera for testing the cross-reactivity of assays; Dr Conrad Quinn and Hanan Dababneh for providing sera from healthy individuals in the USA; and Rebekah Tiller and Dr Marta Guerra for a critical review of the manuscript. Samples from patients in the USA were collected in a previous study and were later transferred to the CDC; the use of samples was approved by the CDC Institutional Review Board (no. 5343). The use of sera from healthy individuals was approved by CDC IRB (no. 3344). Samples from Egypt were collected by NAMRU-3 (NAMRU-3 IRB no. NAMRU3.1999.0001CR09-CONV-M) during an AFI surveillance study. For these samples, no contact was made with human subjects by the CDC, and the CDC did not receive any identifiable information; therefore, the CDC IRB determined that the research did not involve human subjects, as defined under 45 CFR 46.102(h), and an additional IRB review was not required. This research was supported in part by an appointment to the Emerging Infectious Diseases (EID) Fellowship Program administered by the Association of Public Health Laboratories (APHL) and funded by the CDC. The findings and conclusions presented in this manuscript are those of the authors and do not necessarily represent the official position of the CDC and NAMRU-3.

\section{REFERENCES}

Afifi, S., Earhart, K., Azab, M. A., Youssef, F. G., El Sakka, H., Wasfy, M., Mansour, H., El Oun, S., Rakha, M. \& Mahoney, F. (2005). Hospitalbased surveillance for acute febrile illness in Egypt: a focus on community-acquired bloodstream infections. Am J Trop Med Hyg 73, 392-399.

Al Dahouk, S., Tomaso, H., Nöckler, K., Neubauer, H. \& Frangoulidis, D. (2003). Laboratory-based diagnosis of brucellosis a review of the literature. Part II: serological tests for brucellosis. Clin Lab 49, 577-589.

Araj, G. F. (1999). Human brucellosis: a classical infectious disease with persistent diagnostic challenges. Clin Lab Sci 12, 207-212.

Araj, G. F., Lulu, A. R., Mustafa, M. Y. \& Khateeb, M. I. (1986a). Evaluation of ELISA in the diagnosis of acute and chronic brucellosis in human beings. J Hyg (Lond) 97, 457-469.

Araj, G. F., Lulu, A. R., Saadah, M. A., Mousa, A. M., Strannegard, I. L. \& Shakir, R. A. (1986b). Rapid diagnosis of central nervous system brucellosis by ELISA. J Neuroimmunol 12, 173-182.

Araj, G. F., Dhar, R., Lastimoza, J. L. \& Haj, M. (1990). Indirect fluorescent antibody test versus enzyme-linked immunosorbent assay and agglutination tests in the serodiagnosis of patients with brucellosis. Serodiag Immunother Infect Dis 4, 1-8.

Bajani, M. D., Ashford, D. A., Bragg, S. L., Woods, C. W., Aye, T., Spiegel, R. A., Plikaytis, B. D., Perkins, B. A., Phelan, M. \& other authors (2003). Evaluation of four commercially available rapid serologic tests for diagnosis of leptospirosis. J Clin Microbiol 41, 803809.

Brown, S. L., Klein, G. C., McKinney, F. T. \& Jones, W. L. (1981). Safranin O-stained antigen microagglutination test for detection of brucella antibodies. J Clin Microbiol 13, 398-400.

Buchanan, T. M. \& Faber, L. C. (1980). 2-Mercaptoethanol brucella agglutination test: usefulness for predicting recovery from brucellosis. J Clin Microbiol 11, 691-693. 
Cakan, G., Bezirci, F. B., Kacka, A., Cesur, S., Aksaray, S., Tezeren, D., Saka, D. \& Ahmed, K. (2008). Assessment of diagnostic enzyme-linked immunosorbent assay kit and serological markers in human brucellosis. Jpn J Infect Dis 61, 366-370.

CDC (1997). Case definitions for infectious conditions under public health surveillance. MMWR Recomm Rep 46, 1-55.

Fleiss, J. L. (1981). Statistical Methods for Rates and Proportions. New York: John Wiley \& Sons.

Gotuzzo, E., Carrillo, C., Guerra, J. \& Llosa, L. (1986). An evaluation of diagnostic methods for brucellosis - the value of bone marrow culture. J Infect Dis 153, 122-125.

Mantecón, M. A., Gutiérrez, P., Del Pilar Zarzosa, M., Dueñas, A. I., Solera, J., Fernández-Lago, L., Vizcaíno, N., Almaraz, A., Bratos, M. A. \& other authors (2006). Utility of an immunocaptureagglutination test and an enzyme-linked immunosorbent assay test against cytosolic proteins from Brucella melitensis B115 in the diagnosis and follow-up of human acute brucellosis. Diagn Microbiol Infect Dis 55, 27-35.
Marrodan, T., Nenova-Poliakova, R., Rubio, M., Ariza, J., Clavijo, E., Smits, H. L. \& Diaz, R. (2001). Evaluation of three methods to measure anti-Brucella IgM antibodies and interference of IgA in the interpretation of mercaptan-based tests. J Med Microbiol 50, 663666.

Pappas, G., Akritidis, N., Bosilkovski, M. \& Tsianos, E. (2005). Brucellosis. N Engl J Med 352, 2325-2336.

Sharma, R., Chisnall, C. \& Cooke, R. P. (2008). Evaluation of inhouse and commercial immunoassays for the sero-diagnosis of brucellosis in a non-endemic low prevalence population. J Infect 56, 108-113.

Sim, J. \& Wright, C. C. (2005). The kappa statistic in reliability studies: use, interpretation, and sample size requirements. Phys Ther 85, 257268.

Young, E. J. (1995). An overview of human brucellosis. Clin Infect Dis 21, 283-289.

Young, E. J. \& Corbel, M. J. (1989). Brucellosis: Clinical and Laboratory Aspects. Boca Raton, FL: CRC Press. 